\title{
Acknowledgement to Reviewers of Cells in 2015
}

\author{
Cells Editorial Office, \\ Published: 27 January 2016 \\ MDPI AG, Klybeckstrasse 64, CH-4057 Basel, Switzerland; cells@mdpi.com
}

The editors of Cells would like to express their sincere gratitude to the following reviewers for assessing manuscripts in 2015.

We greatly appreciate the contribution of expert reviewers, which is crucial to the journal's editorial decision-making process. Several steps have been taken in 2015 to thank and acknowledge reviewers. Good, timely reviews are rewarded with a discount off their next MDPI publication. By creating an account on the submission system, reviewers can access details of their past reviews, see the comments of other reviewers, and download a letter of acknowledgement for their records. In addition, MDPI has launched a collaboration with Publons, a website that seeks to publicly acknowledge reviewers on a per journal basis. This is all done, of course, within the constraints of reviewer confidentiality. Feedback from reviewers shows that most see their task as a voluntary and mostly unseen work in service to the scientific community. We are grateful to our reviewers for the contribution they make.

\begin{tabular}{lll} 
Adhikari, Neeta & Kamei, Caramai N. & Santillo, Michael \\
Agarwal, Saurabh & Kaneshiro, Edna & Santulli, Gaetano \\
Alugubelly, Navatha & Keane, Niamh M. & Satir, Peter \\
Anderson, Robert & Ki, Sung Hwan & Sazer, Shelley \\
Anderson, Kenneth C. & Kutay, Ulrike & Scharf, Andrea \\
Anthony, Donald & Langenhan, Tobias & Schlatter, Eberhard \\
Aspenström, Pontus & Langer, Rupert & Secchi, Maria Francesca \\
Ayrault, Olivier & Lavia, Patrizia & Seluanov, Andrei \\
Bank, Ruud A. & Li, Zhiping & Sengupta, Debrup \\
Bastin, Philippe & Li, Jinhua & Smith, Jason G. \\
Bazzaro, Martina & Lopes, Susana & Sterkers, Yvon \\
Beltran, William A. & Madura, Kiran & Sussman, Mark A. \\
Bergmann, Carsten & Mahal, Katharina & Terhorst, Cox \\
Boletta, Alessandra & Martinez-Vicente, M. & Tezapsidis, Nikolaos \\
Calvet, James & Maruyama, Toru & Tooze, Sharon A. \\
Céline, Masclaux-Daubresse & Matchett, Kyle B. & Torres, Vicente E. \\
Chen, Xingzhen & Medalia, Ohad & Tran, Pamela \\
Ciarimboli, Giuliano & Mitra, Abhisek & Trisciuoglio, Daniela \\
Corda, Daniela & Momoi, Takashi & Tubaro, Aurelia \\
Costantini, Frank & Montini, Giovanni & Tucker, Kerry L. \\
Davidson, Alan & Montpetit, Ben & Vaughan, Sue \\
Davies, Jamie A. & Moretti, Matteo & Vejux, Anne \\
\hline
\end{tabular}


De Pinto, Vito

Dikalov, Sergey

Elena N., Pugacheva

Ferrari, Luca

Gillette, Thomas

Goswami, A

Goumans, Marie-Josè

Gray, Phillip N.

$\mathrm{Gu}$, Yan

Harel, Amnon

Hori, Manabu

Hung, Mien-Chie

Imamoto, Naoko

Jackson, Claire L.

Jalkanen, Sirpa

Jans, David

Jansen, Gert
Mukhopadhyay, S.

Müller, Roman-Ulrich

Nauli, Surya M.

Nozu, Kandai

Obara, Tomoko

Özdinler, P. Hande

Pantziarka, Pan

Pazour, Gregory J.

Petersen-Jones, Simon

Petit, Patrice X.

Plaza-Díaz, Julio

Prakash, Siddharth K.

Rivero, Francisco

Rossini, Gian Paolo

Rottembourg, Diane

Roy, Nilotpal

Sánchez-Madrid, Francisco
Vogel, Arndt

Wakamiya, Nobutaka

Wallace, Darren P.

Wang, Ying-Jan

Weiss, Thomas $\mathrm{S}$.

Wheway, G.

Wickramasinghe, Vihandha O.

Wilkinson, Kim

Wingert, Rebecca A.

Wolfrum, Uwe

$\mathrm{Xu}$, Pengfei

Yamada, Gen

Yamamoto, Janet

Yamashita, Michiaki

Zhang, Qing

Zhou, Weibin

Zhou, Jing

(C) 2016 by the authors; licensee MDPI, Basel, Switzerland. This article is an open access article distributed under the terms and conditions of the Creative Commons by Attribution (CC-BY) license (http://creativecommons.org/licenses/by/4.0/). 